\title{
Postconditioning CS-alone exposure as a source of interference in a taste aversion paradigm
}

\author{
STEPHEN B. KLEIN, PETER J. MIKULKA, FENDELL P. ROCHELLE, \\ and VICKIE BLAIR \\ Old Dominion University, Norfolk, Virginia 23508
}

\begin{abstract}
Two studies were conducted to study the effects of unpaired sucrose (CS) experiences on the acquisition, retention, and reacquisition of a sucrose aversion. In Experiment 1, rats received 0,1 , or 3 preconditioning CS exposures and/or 0,1 , or 3 postconditioning CS exposures. Sucrose exposures prior to conditioning interfered with the acquisition of a sucrose aversion, while sucrose-only experience following a single taste-illness pairing retroactively interfered with retention of the sucrose aversion and proactively interfered with the reacquisition of the sucrose aversion. In Experiment 2, two unpaired sucrose experiences following each sucroseillness pairing (33\% PRF group) resulted in little apparent sucrose aversion after six sucroseillness pairings, while a single unpaired sucrose experience after each conditioning trial $150 \%$ PRF group) produced a moderate level of interference compared to the rapid acquisition in rats receiving only sucrose-illness pairings (CRF group). It is suggested that CS familiarization and extinction may represent a single process where the rat learns that a taste cue predicts no illness, and that this expectancy can interfere with the development of an aversion or impair the ability of the animal to reacquire a previously acquired taste aversion.
\end{abstract}

The novelty of a stimulus has been shown to be an important factor in the establishment of a conditioned taste aversion. Exposure to a taste prior to its pairing with illness retards the development of the aversion (Elkins, 1973; Fenwick, Mikulka, \& Klein, 1975; McLaurin, Farley, \& Scarborough, 1963; Mikulka \& Klein, 1977). Also, rats will rapidly develop an aversion to a novel taste even when both the novel and familiar tastes are paired with illness (Kalat, 1974; Kalat \& Rozin, 1973; Revusky \& Bedarf, 1967). Interpretations of the CS preexposure effects have suggested that the rat learns that a taste can be safely consumed (Kalat \& Rozin, 1973) or is irrelevant (MacKintosh, 1973), or that latent inhibition develops (Best, 1975).

Recently, a number of studies have reported a similar interference with the acquisition of a taste aversion following the presentation of illness (US) experiences without any accompanying taste cue (i.e., Berman \& Cannon, 1974; Braveman, 1975; Cannon, Berman, Baker, \& Atkinson, 1975; Cappell \& LeBlanc, 1975; Elkins, 1974; Mikulka, Leard, \& Klein, 1977; Parker, Failor, \& Weidman, 1973; Riley, Jacobs, \& Lolordo, 1976; Gamzu, Note 1; Vogel \& Nathan, Note 2). Mikulka et al. (1977) reported that postconditioning exposures to the US alone can also retroactively attenuate the strength of the original taste aversion. Their results indicated that the amount of

Reprint requests should be sent to Dr. Stephen B. Klein, Psychology Department, Old Dominion University, Norfolk, Virginia 23508 . retroactive interference was dependent upon the number of US alone exposures (i.e., six US-alone exposures produced more retroactive interference than two US-alone experiences). A similar study by Riley et al. (1976) found that postconditioning US-alone exposures interfered with the subsequent development of a second taste aversion, although they failed to find a retroactive effect of US-alone experience.

The first experiment was designed to investigate the influence of unpaired postconditioning CS exposures on the retention and reacquisition of a prior taste aversion. Both US- and CS-alone exposures have been shown to proactively interfere with the acquisition of a taste aversion, while postconditioning US-alone exposures retroactively interfere with the retention of a previously acquired aversion and proactively impaired the development of a new aversion. An information value model of conditioning (based on Rescorla's, 1968, contingency data) would suggest that unpaired postconditioning CS or US experiences would interfere with the retention and reacquisition of a CS-US association. Thus, it was anticipated that postconditioning CS-alone exposures would have an effect similar to that seen with postconditioning US-alone exposures, and therefore, would interfere with the retention and reacquisition of a previously acquired taste aversion.

\section{EXPERIMENT 1}

Rats received zero, one, or three unpaired sucrose 
exposures prior to a single sucrose- $\mathrm{LiCl}$ pairing in Experiment 1. Following the taste-illness pairing, an equal number of rats from each preexposure group received zero, one, or three unpaired CS-alone exposures. After this, taste-illness pairings were continued until a suppression criterion was reached.

\section{Method}

Subjects. The subjects were 54 naive female rats purchased from Sprague-Dawley (Madison, Wisconsin). The rats were 75 days old and weighed approximately $200 \mathrm{~g}$ at the start of this experiment. All rats were received in the laboratory 7 days prior to the start of this study.

Procedure. Each subject was placed on a 23.5-h water-deprivation schedule for 3 days prior to the beginning of the experiment. All fluids were presented in the home cage at approximately the same time each day throughout the entire experiment. The amount of water consumed in $30 \mathrm{~min}$ on the last water-deprivation day was recorded for each subject. All rats consumed at least $10 \mathrm{~g}$ of solution on the last deprivation day.

Following the 3 water-deprivation days, the subjects were randomly divided into three experimental groups. These groups differed in terms of the treatment received in Phase 1. Animals in the 0 -preexposure group $(n=18)$ received 3 additional days of 30 -min water access during Phase 1. Animals in the 1-preexposure group $(n=18)$ were given a single 30 -min exposure to sucrose during Phase 1. On Day 2 of Phase 1, the 1-preexposure animals received 30-min access to $10 \%$ sucrose, while on Days 1 and 3 of Phase 1 water was available for $30 \mathrm{~min}$. Animals in the 3-preexposure group $(n=18)$ were given 30 -min access to sucrose on Days 1, 2, and 3. In an attempt to maintain constant fluid intake, water was made available immediately following sucrose access in an amount equal to the water intake on the last deprivation minus the amount of sucrose consumed on the days the animals had access to sucrose.

On Day 1 of Phase 2, sucrose was available for $30 \mathrm{~min}$ for all rats. Following sucrose exposure, all subjects were given a IP $\mathrm{LiCl}$ injection $(3 \mathrm{mgEq} / \mathrm{kg}$ dosage using a $.6 \mathrm{M} \mathrm{LiCl}$ solution of $.5 \%$ body weight). ${ }^{1}$ An equal number of rats $(n=6)$ from each preexposure condition received one of three treatments during Phase 2. Animals in the 0-postconditioning exposure group were given 30 min of water access during Days 2,3 , and 4 of Phase 2 . The 1-postconditioning exposure animals received a 30 -min exposure to sucrose on Day 3 of Phase 2 and were given water for $30 \mathrm{~min}$ on Days 2 and 4 . Sucrose was available for $30 \mathrm{~min}$ on Days 2, 3, and 4 of Phase 2 for all animals in the 3-postconditioning exposure group. This 3 by 3 factorial design varied three levels of preconditioning sucrose exposure and three levels of postconditioning exposure. This yielded nine groups: 0 Pre-0 Post, 0 Pre-1 Post, 0 Pre-3 Post, 1 Pre-0 Post, 1 Pre-1 Post, 1 Pre-3 Post, 3 Pre- 0 Post, 3 Pre- 1 Post, 3 Pre- 3 Post (the first term designates the number of preconditioning sucrose experiences, and the second term shows the number of postconditioning sucrose exposures).

During Phase 3, all subjects received 30-min access to sucrose followed by a $\mathrm{LiCl}$ injection. Sucrose access and $\mathrm{LiCl}$ injections were given every other day until sucrose consumption dropped to $1 \mathrm{~g}$ or less on any given day. Water was available $1 \mathrm{~h}$ after $\mathrm{LiCl}$ injection in an amount equal to the water intake on the last deprivation day minus sucrose intake on injection days. As in previous phases on noninjection days, water was available for $30 \mathrm{~min}$

For analysis and presentation of the results, Conditioning Days 1-5 refer to sucrose intake on injection days during Phases 2 and 3. The dependent measures of sucrose and water intake obtained prior to and during conditioning were analyzed by analyses of variance. Duncan's multiple range tests (Edwards, 1960) were employed to compare the treatment means $(p<.05$ used throughout).

\section{Results and Discussion}

Statistical analysis of water intakes on the last water-only day of preexposure indicated that the nine experimental groups were not significantly different, with an overall mean intake of $11.2 \mathrm{ml}$ of water. Also, analysis of water intake on the day following the second sucrose-illness pairing found no significant differences between groups in terms of water intake, with consumption being uniformly high in all rats (overall $\bar{X}=15.0$ ). A direct difference $t$ test between the water intake prior to the first conditioning trial and after the second sucrose-illness pairing indicated that the water intake on nonillness days increased over the training period $(p<.01)$. In addition, all groups drank a uniformly high amount of water on the day following the second sucrose-illness pairing. It appears that no aversion generalized to water during conditioning. The observed high water intakes in all groups on days when only water was available persisted throughout the experiment. At no time during the experiment did rats fail to consume water when available and in sufficient level to maintain a constant fluid intake (an increase in water intake compensated for a decrease in sucrose intake).

Figure 1 presents sucrose intake during Conditioning Days 1-5 as a function of number of preconditioning and postconditioning sucrose-alone experiences. The results demonstrated that prior exposure to sucrose did not influence sucrose intake on the first conditioning day and that all groups were equal prior to postconditioning sucrose exposures. Postconditioning CS-alone exposures produced higher intakes during Days 2 and 3 of conditioning than was observed in animals not receiving postconditioning CS-alone exposures. In addition, sucrose intakes were not

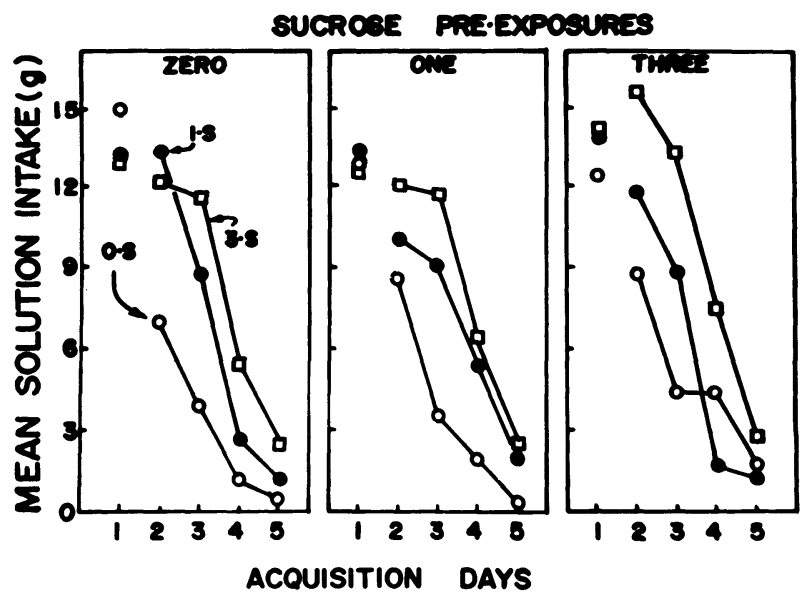

Figure 1. Mean sucrose solution intake during 5 aequisition days as function of number of $\mathrm{CS}$ pre- and postconditioning days. Open circles represent 0 postconditioning $C S$ exposures, closed circles represent 1 postconditioning $C S$ exposure, and open squares represent 3 postconditioning $C S$ experiences. The first panel presents the 0 - preconditioning CS groups, the second panel presents the 1-preconditioning CS group, and the third panel presents the 3-preconditioning $\mathrm{CS}$ groups. 
affected by the number of preconditioning sucrose exposures. These conclusions were based on the results of a 3 (Pre) by 3 (Post) by 5 (Days) analysis of variance. This analysis yielded a significant postconditioning CS effect $[\mathrm{F}(2,45)=11.19, \mathrm{p}<.005]$, a significant Days effect $[F(4,184)=138.76, p<.05]$, and a significant Postconditioning CS by Days interaction $[F(8,184)=5.50, p<.005]$. All other effects were nonsignificant $(F<1)$. Subsequent post hoc comparisons showed that Day 1 intakes were equivalent for all groups, while Day 2 sucrose intakes were significantly higher in subjects that had either one or three postexposures than was observed in the 0sucrose postexposure groups. Comparison of Conditioning Days 3 and 4 showed that the 3 -postconditioning CS groups drank significantly more sucrose than the 0 -postconditioning $C S$ group rats. The intakes of the 1-postconditioning CS groups were intermediate between the 0- and 3-postconditioning CS groups. By Day 5 of conditioning, there were no observed differences in sucrose intake. These results indicate that postconditioning CS exposures interfered with the retention of a prior sucrose-illness association.

One might expect that post exposures would extinguish the prior taste-illness association and that the subject would then reacquire the aversion as rapidly or more rapidly than the naive subjects. However, in this study, postconditioning sucrose exposures interfered with the reacquisition of the sucrose aversion. To clearly ilustrate this effect, the acquisition of four groups was compared: The naive subjects ( 0 Pre- 0 Post), the CS familiarized subjects (3 Pre-0 Post), the subject receiving only postconditioning CS exposure (0 Pre-3 Post), and those receiving both pre- and postconditioning CS exposures (3 Pre-3 Post). For statistical comparison, the acquisition analyses compared the development of the taste aversion for the first two groups, using their data beginning with their first CS-US pairing, while the data for the second groups began with the first CS-US pair after their 3 postconditioning exposures (actually their second CS-US pairing). Figure 2 presents this comparison, with the four groups lined up at their relative acquisition starting points. This procedure allows a reasonable assessment of the effects of pre- and postconditioning CS exposure on the development of a taste aversion. The asterisk refers to the initial conditioning day intakes for the 0 Pre-3 Post and 3 Pre-3 Post groups. The 0 Pre-3 Post rats consumed more sucrose during relearning than did 0 Pre-0 Post animals. In contrast, preconditioning sucrose exposures only marginally interfered with the development of the aversion, producing significantly less interference than was observed following postconditioning CS exposures. The combination of pre- and postsucrose exposures appeared to pro- duce the most durable interference. In support of these conclusions, a 4 (Groups) by 4 (Days) analysis of variance yielded a nonsignificant Groups effect $[F(3,20)=1.97]$, a significant Days effect $[F(3,61)$ $=77.50, \mathrm{p}<.005$ ], and a significant Groups by Days interaction $[\mathrm{F}(9,61)=2.07, \mathrm{p}<.05]$. Multiple comparisons of the data showed that intake of sucrose on Day 1 was significantly higher in the 3 Pre-3 Post than in the 3 Pre- 0 Post and 0 Pre-3 Post rats, which did not differ. Also, the 0 Pre- 0 Post rats tended to drink more sucrose than the 3 Pre-0 Post and the 0 Pre-3 Post rats $(.05<\mathrm{p}<.10)$, while sucrose intake in the 3 Pre- 3 Post and the 0 Pre-0 Post was not different on Day 1. The 3 Pre-3 Post group animals exhibited significantly higher sucrose intakes on Days 2 and 3 than the 0 Pre- 0 Post rats, while the 0 Pre-3 Post animals drank more sucrose than the 0 Pre-0 Post animals on Day 2. Sucrose intake in the 3 Pre- 0 Post rats was greater than the 0 Pre-0 Post rats on Day $2(.05<p<.10)$ and Day 4 , while being significantly lower than the 3 Pre-3 Post rats on Days 2 and 3 and the 0 Pre-3 Post rats on Day 2. The 3 Pre-3 Post and the 0 Pre-3 Post animals were not different over Days 2-4.

The major result of Experiment 1 was that postconditioning sucrose exposure reduced the organism's aversion to sucrose and subsequently interfered with the reacquisition of the sucrose aversion. This postconditioning interference was similar to that observed when a taste experience preceded the original acquisition, although in this study only a weak familiarization effect was found. However, a combination of pre- and postconditioning sucrose exposures produced the greatest level of reacquisition interference.

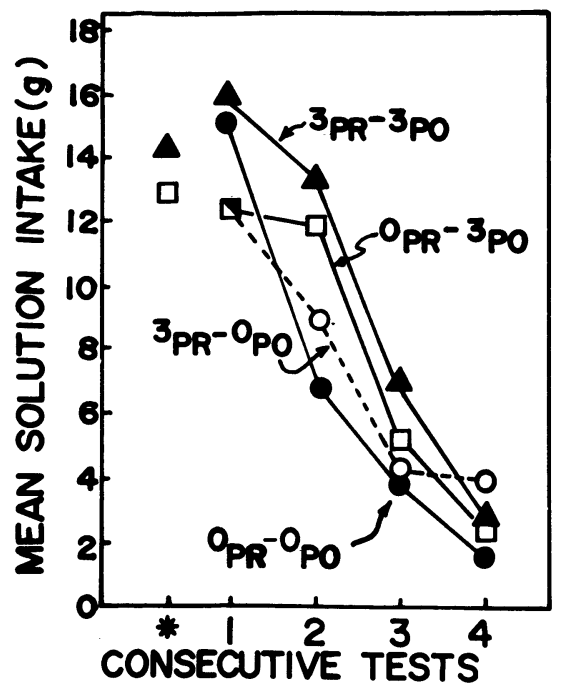

Figure 2. Mean sucrose solution intake during the 4 acquisition days for the 3 pre- 0 postconditioning and the 0 pre-0 postconditioning groups, and the initial acquisition (indicated by the asterisk) and the 4 reacquisition days in the 0 pre-3 postconditioning and the 3 pre-3 postconditioning groups. 
In many situations, the reacquisition of a response is more rapid than original acquisition (Pavlov, 1927). This rapid reacquisition may be due to the contribution of nonspecific positive transfer outweighing negative transfer effects. Apparently, in the present taste aversion situation, the negative transfer from extinction was the stronger factor, and post-CS exposures proactively interfered with the reacquisition of a taste aversion. Whether interference in flavor aversion situations operates differently from other learning paradigms should be the focus of future research.

\section{EXPERIMENT 2}

The results of Experiment 1 indicated that exposure to the CS after a single conditioning trial interfered with the development of the taste aversion. The strong postconditioning CS effect suggests that there may be conditions under which post-CS exposures may prevent the development of a taste aversion. This is all the more surprising, given the rapidity of taste-illness learning in rats. The purpose of this experiment was to determine to what extent the presentation of CS-alone experiences after each CS-illness pairing would interfere with the acquisition of sucrose-illness association. Either one or two sucrose exposures were given after each sucrose-illness conditioning trial.

\section{Method}

Subjects. The subjects were 21 naive female rats similar to those used in the first experiment.

Procedure. Each subject was placed on a 23.5-h water-deprivation schedule for 7 days prior to the beginning of the experiment. The amount of water consumed during the 30 -min session on the last water-deprivation day was recorded for each subject. No animal consumed less than $12 \mathrm{~g}$ of solution on the last deprivation day prior to beginning of Experiment 2.

On the next day, all subjects received 15 -min access to a $10 \%$ sucrose solution followed by $15 \mathrm{~min}$ of water and then a $\mathrm{LiCl}$ injection. Sucrose-illness pairings occurred every 5 th day until the amount consumed was less than $1 \mathrm{~g}$. This procedure was identical to the method used in Klein, Domato, Hallstead, Stephens, and Mikulka (1975), which readily established a taste aversion to sucrose.

Animals in the CRF group $(n=7)$ received 30 min of water on the 4 intervening days between sucrose-illness pairings, while the PRF 50 animals $(n=6)$ were given $15 \mathrm{~min}$ of $10 \%$ sucrose followed by 15 min of $\mathrm{H}_{2} \mathrm{O}$ on the 3rd day after each sucroseillness pairing. The animals in PRF 33 group $(n=7)$ received $15 \mathrm{~min}$ of sucrose and $15 \mathrm{~min}$ of water on the $3 \mathrm{rd}$ and 4 th day following each sucrose-illness pairing. Water was available for $30 \mathrm{~min}$ on other nonillness days.

\section{Results}

Prior to conditioning (on the last water-deprivation day), all groups were statistically equivalent in their intakes $(\bar{X}=15.8)$. Statistical analysis showed no change in water intake over the course of the study. Water consumption remained high throughout the entire experiment $(\overline{\mathrm{X}}=13.8$ after first conditioning trial, 16.0 after third conditioning trial, 14.5 after fifth conditioning trial). Thus, the intake of water was equivalent in each treatment condition on noninjection days. Additionally, the subjects consumed enough water to maintain approximately a constant daily fluid intake (an increase in water consumption offset any decreased in sucrose intake) on injection days.

Figure 3 presents the mean sucrose solution intake over the 7 conditioning days for the CRF, PRF 50, and PRF 33 animals. The acquisition of sucrose aversion was significantly impaired by the presentation of postconditioning.sucrose exposures after each conditioning trial, with the weakest aversion developing when two postconditioning taste-alone exposures followed each taste-illness pairing. A repeated measures analysis of variance was conducted on the sucrose intakes over the 7 days of conditioning and yielded a significant Groups effect $[\mathrm{F}(2,18)=30.58$, $\mathrm{p}<.001]$, a significant Days effect $[\mathrm{F}(6,108)=91.35$, $\mathrm{p}<.001$, and a significant Groups by Days interaction $[F(12,108)=5.18, p<.001]$. Range tests indicated that sucrose intakes were equal on the 1 st conditioning day, while the PRF 33 and PRF 50 subjects drank more sucrose on Days 2 through 7 than did the CRF subjects. In addition, intake in PRF 33 was consistently higher on Conditioning Days 2-7 than was observed in PRF 50 rats. Although the CS exposures after each conditioning trial did markedly attenuate the development of the taste aversion, sucrose intakes of all the animals on Day 7 were significantly below the level observed on Day 1 of conditioning. After six conditioning trials, sucrose intake in the PRF 50 and PRF 33 groups had decreased $75 \%$ and $25 \%$, respectively, from their initial levels.

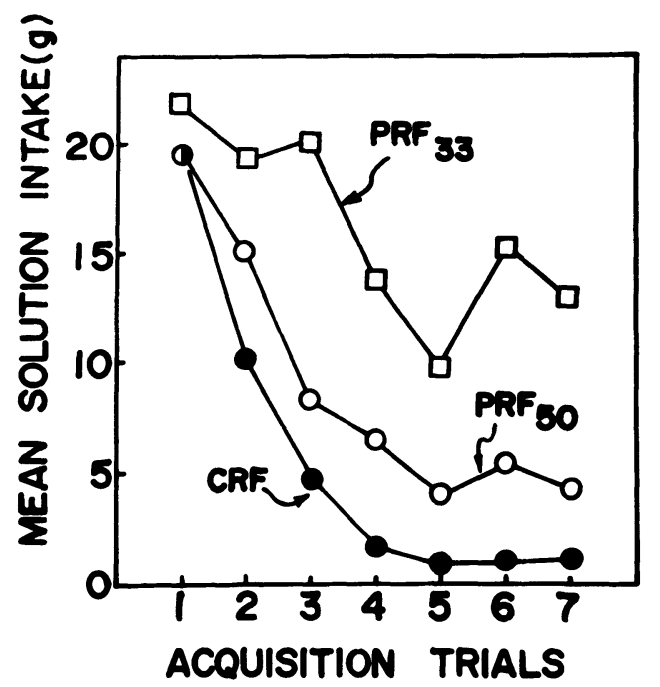

Figure 3. Mean sucrose solution intake during the 7 acquisition days for the CRF, PRF 50\%, and PRF 33\% groups of Experiment 2. 


\section{GENERAL DISCUSSION}

The present research has shown that if a rat experiences a sucrose taste without any associated illness, this CS-alone experience interferes with the acquisition, retention, and reacquisition of a sucroseillness pairing. Although exposure to sucrose prior to conditioning produced only a minimal interference effect in Experiment 1, the literature has consistently reported CS familiarization effects on taste-aversion conditioning (Elkins, 1973; Fenwick et al., 1975; McLaurin et al., 1963; Mikulka \& Klein, 1977).

The results of both experiments clearly demonstrated that taste exposures (CS alone) after conditioning interfered with the retention of the prior sucrose-illness association. Initial sucrose aversions were significantly stronger in rats not given sucrose after a single conditioning trial than in rats receiving either one or three postconditioning sucrose-alone experiences. In addition, the presentation of sucrose after conditioning impaired the reacquisition of a taste aversion, while subjects given three CS exposures after a single conditioning trial in Experiment 1 took significantly longer to reacquire their aversion to sucrose than did subjects that received no post-CS experiences or rats receiving three preconditioning sucrose exposures. Thèse results suggest that postconditioning exposure to a CS did not merely weaken the prior taste-illness association, but rather interfered with the reappearance of the taste aversion. The power of postconditioning CS exposure was demonstrated in Experiment 2. After six taste-illness pairings, the sucrose intake in rats receiving two CSalone exposures after each trial was approximately $75 \%$ of the preconditioning level (Trial 1). In contrast, animals given water on the intervening days showed a greater sucrose suppression after only a single trial $(55 \%)$. It is apparent that postconditioning CS exposures severely disrupted the development of the sucrose aversion.

This postconditioning CS interference with the retention and reappearance of a taste aversion is similar to that observed with postconditioning US exposures (Mikulka et al., 1977). They reported that illness-alone experiences given after conditioning interfered with the retention of a prior taste-illness association, while Riley, Jacobs, and Lolordo (1976) reported that post-US-alone exposures interfered with the acquisition of a new taste aversion. A similar postconditioning US exposure effect has been reported using loud noise and shock (Rescorla, 1973, 1974). One explanation for the effect of postconditioning US exposure is that the organism learns that the US occurs independently of the taste cue (a nontasteillness expectancy) and this expectancy interferes with the retention of a prior taste-illness expectancy. The results of Experiments 1 and 2 as well as those of Mikulka et al. (1977) indicate that experience with either the CS or the US after conditioning interferes with retention and relearning of the prior taste-illness association.

A parsimonious explanation for these data is that the same process accounts for both CS and US interference. It may be that the animal learns during conditioning that a taste cue predicts illness. As a consequence of either post conditoning CS-alone or US-alone exposure, the rat learns that the CS (taste) does not reliably predict illness or that US (illness) is not reliably predicted by a taste cue. Further, it is suggested that preconditioning CS or US exposures interfere with development of a taste-illness association in the same manner as do postexposures. During prior CS exposures, the rats develop an expectancy that the CS does not predict illness, while prior US exposures produce a nontaste-illness association (US does not have any predicting taste cues). These expectancies can interfere with development, retention, or reacquisition of a taste aversion (taste-illness association). This conceptualization of interference is similar to that used in verbal learning situations (Spear, 1970).

The observation that CS familiarization and extinction represent identical processes is not new and was discussed in the context of fear conditioning by Leaf, Kayser, Andrews, Adkins, and Leaf (1968). Leaf et al. (1968) observed that an equivalent amount of fear reduction, as measured by drinking suppression, occurred when nonreinforced CS exposures either preceded (habituation) or followed (extinction) conditioning. They suggested that habituation might be functionally, in addition to being operationally, similar to extinction. However, the results in this study provide only suggestive support for an equivalence of habituation (preconditioning CS exposure) and extinction (postconditioning CS exposure), since the degree of pre- and postexposures interference was not equivalent. Future research should focus on determining what the influence is on extinction (or habituation) of variables known to habituation (or extinction).

\section{REFERENCE NOTES}

1. Gamzu, E. Pre-exposure to an unconditioned stimulus alone may eliminate taste aversion. Paper presented at the 15th Annual Psychonomic Society meeting, Boston, November 1974.

2. Vogel, J. R., \& Nathan, B. A. Antagonism of a taste aversion by pre-exposure to a dissimilar drug. Paper presented at 83rd Annual Meeting of the American Psychological Association, Chicago, September 1975.

\section{REFERENCES}

Berman, R. F., \& Cannon, D. S. The effect of prior ethanol experience on ethanol-induced saccharin aversion. Physiology and Behavior, 1974, 12, 1041-1044.

BEST, M. Conditioned and latent inhibition in taste-aversion 
learning: Clarifying the role of learned safety. Journal of Experimental Psychology: Animal Behavior Processes, 1975, 104. $97-113$.

Braveman, N. S. Formation of taste aversions in rats following prior exposure to sickness. Learning and Motivation, 1975, 6. 512-534.

Cannon, D., Berman, R., Baker, T., \& Atrinson, C. Effect of preconditioning unconditioned stimulus experience on learned taste aversions. Journal of Experimental Psychology: Animal Behavior Processes, 1975, 104, 270-284.

Cappell, H. D., \& LeBlanc, A. E. Conditioned aversion by amphetamine: Rates of acquisition and loss of the attenuating effects of prior exposure. Psychopharmacologia, 1975, 43, 157-162.

EDWARDs, H. L. Experimental design in psychological research (3rd ed.). New York: Holt, Rinehart, \& Winston, 1960.

Elkins, R. L. Attenuation of drug induced bait shyness to a palatable solution as an increasing function of its availability prior to conditioning. Behavioral Biology, 1973, 9, 221-226.

ElKINS, R. L. Bait shyness acquisition and resistance to extinction as functions of US exposure prior to conditioning. Physiological Psychology, 1974, 2, 341-343.

Fenwick, S., Mikulka, P. J., \& Klein, S. B. The effect of preexposure to sucrose on the acquisition and extinction of a conditioned aversion. Behavioral Biology, 1975, 14, 231-235.

KALAT, J. M. Taste salience depends on novelty, not concentration, in taste aversion learning in the rat. Journal of Comparative and Physiological Psychology, 1974, 86, 47-50.

Kalat, J. W., \& Rozin, P. "Learned safety" as a mechanism in long-delay taste-aversion learning in rats. Journal of Comparative and Physiological Psychology, 1973, 83, 198-207.

Klein, S. B., Domato, G. C., Hallstead, C., Stephens, I., \& Mikulka, P. J. Acquisition of a conditioned aversion as a function of age and measurement technique. Physiological Psychology, 1975, 3, 379-384.

Leaf, R. C., Kayser, R. J., Andrews, J. S., Jr., Adkins, J. W., \& LEAF, S. R. D. Block of fear conditioning induced by habituation or extinction. Psychonomic Science, 1968, 10, 189-190.

MacKintosh. N. J. Stimulus selection: Learning to ignore stimuli that predict no change in reinforcement. In R. A. Hinde \& J. G. Stevenson (Eds.), Constraints on learning: Limitations and predispositions. London: Academic Press, 1973. Pp. $75-100$.

Mclaurin, W. A., Farley, J. A., \& Scarborough, B. B. Inhibitory effects of pre-irradiation saccharin habituation on conditioned avoidance behavior. Radiation Research, 1963, 18, 473-478.
MikulKa, P. J., \& Klein, S. B. The effect of CS familiarization and extinction procedure on the resistance to extinction of a taste aversion. Behavioral Biology, 1977, 19, 518-522.

MikulKa, P. J., Leard, B., \& Klein, S. B. Illness alone (US) exposure as a source of interference with the acquisition and retention of a taste aversion. Journal of Experimental Psychology: Animal Behavior Processes, 1977, 3, 189-201.

Nachman, M., \& Ashe, J. H. Learned taste aversions in rats as a function of dosage, concentration, and route of administration of LiCl. Physiology and Behavior, 1973, 10, 73-78.

Parker, L., Failor, A., \& Weidman, K. Conditioned preferences in the rat with an unnatural need state: Morphine withdrawal. Journal of Comparative and Physiological Psychology, 1973, 82, 294-300.

Pavlov, I. P. Conditioned reflexes. New York: Dover, 1960. (Original published in English, 1927.)

Rescorla, R. A. Probability of shock in the presence and absence of CS in fear conditioning. Journal of Comparative and Physiological Psychology, 1968, 66, 1-5.

Rescorla, R. A. Effect of inflation of the unconditioned stimulus value following conditioning. Journal of Comparative and Physiological Psychology, 1974, 86, 101-106.

ResCorla, R. A. Effect of US habituation following conditioning. Journal of Comparative and Physiological Psychology, 1973, 82, 137-143.

Revusky, S., \& Bedarf, W. E. Association of illness with prior ingestion of novel foods. Science, 1967, 155, 219-220.

Riley, A. L., Jacobs, W. J., \& Lolordo, V. M. Drug exposure and the acquisition and retention of a taste aversion. Journal of Comparative and Physiological Psychology, 1976, 90. 799-807.

SPEAR, N. E. Verbal learning and retention. In M. R. D'Amato (Ed.), Experimental psychology: Methodology, psychophysics and learning. New York: McGraw-Hill, 1970.

\section{NOTE}

1. Nachman and Ashe (1973) have demonstrated that taste aversion in rats is dependent upon the equivalent-molar dosage $(\mathrm{mEq} / \mathrm{kg})$ of $\mathrm{LiCl}$, and not on the concentration or volume of $\mathrm{LiCl}$. The dosage employed in the present study was $3.0 \mathrm{mEq} / \mathrm{kg}$, identical to that reported by Nachman and Ashe (1973) to produce a very strong taste aversion in rats.

(Received for publication September 26, 1977; revision accepted January 5, 1978.) 